\title{
Geo/Geo/1/N Queue with In-Immune and Immune Service Killing Discipline
}

\author{
Madhu Jain*, G.C. Sharma* and Sapna Chakrawarti** \\ *Institute of Basic Science, Khandari, Agra-282002 (India) \\ **St. John's College, Agra-282002 (India) \\ *madhujain@sancharnet.in \&**sapna_ibs@yahoo.com
}

Abstract. The present investigation studies a discrete time single server queue with both positive and negative arrival streams in accordance with removal of the customer from the end (RCE)-in immune and immune service killing policy. This study is a generalization of the queue with negative customers, wherein only positive customers need a service and negative customers arriving to the system can kill the already present positive customers from any where in the queue, otherwise get lost. The concept of both in-immune and immune service killing are taken into consideration. According to the in-immune killing policy, the negative customer is allowed to kill the most recent positive customer inspite of whether it is in service or not, while the immune service killing discipline suggests that the customer currently being served is immune from killing by the negative arrival. We analyze a queue with geometric arrivals of both positive and negative customers for a finite capacity system. The stationary probability distribution and other performance measures are derived in terms of the generating functions. The results so obtained are validated by the numerical method based on successive over relaxation method (SOR). We have also employed the neurro fuzzy approach for exhibiting the approximate results for various performance measures.

Keywords: G-queue, Discrete -time, Finite capacity, Negative arrival, In-immune and Immune -service killing, Queue size, Neurro-fuzzy technique. 


\section{Introduction}

Queueing theory, which deals with the probabilistic congestion situations, finds enormous applications in the modeling of the complex systems such as computer and communication networks, assembly line, flexible manufacturing systems, etc. In classical queueing models, a queue generally consists of positive customers only. However, in some practical situations, both positive and negative customers come into existence at the service facility. Negative arrivals are used as a control mechanism in many telecommunications and computer network systems and thus enhance the growing interest of several researchers to study the queueing systems with negative customers.

Gelenbe $^{[1]}$ was the first who introduced the negative customer system with a view of modeling a neural network; the queue with negative customers is known as the G-queue in the honour of Gelenbe. A G-queue permits the arrivals of both positive and negative customers. A negative customer does not need service and can remove a customer from somewhere in a queue. The existence of flow of negative customers provides a control mechanism to excessive congestion in the queue. For example in the telecommunication networks in which time is slotted it happens so; likewise in ATM (asynchronous transfer mode) and BISDN (Broadband integrated services digital networks). A practical example of the queues with both the positive and negative customers can be found in computer systems. For example in computer networks flow of virus infection where virus entering a node can be considered as an arrival of negative customer. A virus (negative customer) may originate from outside the network i.e. through floppy disk or may come from another node into the network i.e. electronic mail and can infect one or more files. When there is no virus in computer, then the system can be modeled and analyzed using conventional queueing network with only positive arrivals. The G-network systems have wide variety of applications in the real situations such as in computer systems, telecommunication, manufacturing system, etc. [cf. Harrison and Pitel $\left.{ }^{[2]}\right]$. Gelenbe ${ }^{[3-6]}$ further extended his work to a wide range of applications including the product form queueing network, the triggered customer movement, and neural network, etc. Artalejo ${ }^{[7]}$ made an approach to study the different aspects of queueing theory using the Gelenbe concept of G-networks. A queue with both positive and negative arrivals 
governed by a Markov chain has been analyzed by Shin and $\mathrm{Choi}^{[8]}$. M/GI/1 queues have been studied by Zhu and Zhang ${ }^{[9]}$, in which both positive and negative arrivals require a service. Wang and Zhang ${ }^{[10]}$ studied an unreliable retrial queueing system with negative customers. Recently, Chae et al. ${ }^{[11]}$ focused on GI/Geo/1 queue with negative and positive customers.

The negative customers are characterized by their arrivals as they eliminate the customers from somewhere in a queue according to some specified discipline and do not take any services from the server. This type of system of negative customers is termed as the killing discipline. In other words under this discipline one has to determine how and where the removal of the positive customer has taken place due to negative customers. The RCE killing discipline insists that a customer can only be removed from the end of the queue. Chhakka and Harrison ${ }^{[12]}$ considered the two variants of RCE killing policy, where the most recent positive customers are removed first ( $c f$. Harrison and Pite ${ }^{[13]}$ ). Details about the inclusion of RCE and RCH killing policies can be found in the work of Atenica and Moreno ${ }^{[14]}$. In the present investigation, we are taking into account the $\mathrm{Geo} / \mathrm{Geo} / 1$ queue in which the arrivals of both the positive and negative customers are geometrically distributed.

Discrete time queueing models are gaining more and more interest by the several researchers due to their wide applicability in the real time situations. Bruneel and $\mathrm{Kim}^{[15]}$ investigated the discrete time models for communication system including the ATM services. A discrete time geometric batch arrival queue has been analyzed by Artalejo et al. ${ }^{[16]}$ by taking into account the retrial queues with negative customers as the control mechanism.

Neurro-fuzzy is an emerging soft-computing methodology that is useful in developing approximations for the complex problems and work on the principle of supervised learning. Recently, Ciarmella et al ${ }^{[17]}$ has suggested the fuzzy relational neural net. In this reference, a neurro-fuzzy and queueing based neural network model for traffic signal timing using two-dimensional correlation has been reported in literature by Kaedi et $a .^{[18]}$.

The present study deals with the discrete time queueing model with both positive and negative arrivals following the geometric distribution of arrival pattern. In this investigation we analyze a finite capacity 
$\mathrm{Geo} / \mathrm{Geo} / 1$ queue with two types of arrival streams in accordance with the RCE in-immune and immune servicing policy, respectively. However, as the closed form results are difficult to be obtained, the numerical method based on successive over relaxation (SOR) approach is used to facilitate the numerical results. Further, the results are also approximated using the neurro-fuzzy technique.

The rest of the paper is organized as follows. The model description and notations are given in Section 2. Section 3 describes the Geo/Geo/1 queue with negative customers with RCE service killing discipline using the generating function method for both immune and inimmune cases. In Section 4, various performance measures along with stationary distribution for queue size are given. The effect of several parameters on the system performance is examined numerically in Section 5. Finally, Section 6 concludes the paper with a brief summary.

\section{Model Description}

Consider a discrete time Geo/Geo/1 queue with finite capacity. The two types of customers i.e. the positive and negative customers arrive according to the geometrical processes with probabilities $\mathrm{p}$ and $\mathrm{q}$, respectively. For analytical convenience, it is required to make an assumption regarding the order of arrival policies namely early arrival system (EAS) and late arrival system (LAS). In LAS, the customers are assumed to arrive late during a slot. On the other hand, if the customers are assumed to arrive early during a slot; then it is referred to as EAS. The time axis is divided into intervals of equal length (called slots); the length of the slot is taken to be unity. We assume that arrivals and departures occur at the slot boundaries and therefore they may occur at the same time. At each time slot, the events occur in sequence, an arrival of positive customer followed by an arrival of negative customer (if any) and departure of the customers (if any). The service times are independent and geometrically distributed with probability $\bar{s}$. Furthermore, it has also been assumed that the service can only take place at the slot boundaries and are independent. Thus all the queueing activities take place at the slot boundaries. We consider the system as LAS with immediate arrival. Further, we assume that arrival occurs in the interval $\left(n^{-}, n\right)$, where $n=0,1,2, \ldots$ and a departure occurs in the interval $\left(\mathrm{n}, \mathrm{n}^{+}\right)$, where $\mathrm{n}=0,1,2, \ldots$ 
Let us define the stochastic the process $X_{m}$, such that $\left\{X_{m}, m=0,1, \ldots \ldots, \mathrm{N}\right\}$ is called Markov chain of one dimension with state space $\{0,1,2, \ldots, \mathrm{N}\}$.

Following are the notations used to describe the system dynamics:

N Finite capacity of the system.

$p \quad$ Probability with which a positive customer arrives such that $p=1-\bar{p}$.

$\bar{p} \quad$ Probability with which a positive customer do not arrive such that $\bar{p}=1-p$.

$q \quad$ Probability with which a negative customer arrives s.t. $q=1-\bar{q}$.

$\bar{q} \quad$ Probability with which a negative customer do not arrive s.t. $\bar{q}=1-q$.

$s \quad$ Probability with which a customer do not take its service in a given slot, $s=1-\bar{s}$.

$\bar{s} \quad$ Probability with which a customer takes its service in a given slot, $\bar{s}=1-s$.

$\rho \quad$ Traffic intensity $(\rho=p / \bar{s})$.

$g(z) \quad$ Marginal p.g.f. of the number of customers in the waiting line, when the server is busy.

$G(z) \quad$ Probability generating function for the system size.

$\pi_{i} \quad$ Probability at equilibrium that the queue is in state ' $i$ ', i.e. there are ' $i$ ' number of customers in the queue.

\section{Generating Function and the Stationary Distribution}

In this section, we describe the generating functions and the stationary distribution of the proposed queueing model. Let $\left\{X_{m}, m=0,1, \ldots \ldots, \mathrm{N}\right\}$ be the Markov chain describing the queueing system.

Thus, $\pi_{k}=\lim _{m \rightarrow \infty} P\left[X_{m}=k\right], 0 \leq k \leq N$.

Introducing an auxiliary generating function $g(\mathrm{z})$, s.t. 


$$
g(z)=\sum_{k=1}^{N} \pi_{k} z^{k-1},|z| \leq 1,
$$

The generating function of the stationary distribution is obtained as

$$
G(z)=\pi_{0}+z g(z)
$$

\subsection{RCE In-immune Service Killing Policy}

Consider the RCE- in-immune service killing policy for the proposed model, in which the negative arrival eliminates the positive customer from the end. An arrival of a positive and a negative customer is denoted by the probabilities $\mathrm{p}$ and $\mathrm{q}$ respectively, $\mathrm{s}$.t. $0<p<1,0<q<1$ and $0<s<1$, where $\mathrm{s}$ being the probability that a customer does not conclude its service at a given slot.

Now the transition probabilities are given as follows:

$$
\begin{aligned}
& p_{0,0}=\bar{p}+p q \\
& p_{1,0}=\bar{s} \bar{p}+\bar{s} p q+s \bar{p} q \\
& p_{2,0}=\bar{s} \bar{p} p_{0,1}=p \bar{q} \\
& p_{3,1}=\bar{s} \bar{p} \\
& p_{1,1}=\bar{s} p \bar{q}+s \bar{p} \bar{q}+s p q \\
& p_{2,1}=\bar{s} \bar{p}+\bar{q} p q+s \bar{p} q \\
& p_{k-1, k}=s p \bar{q}, \quad 2 \leq k \leq N-2 \\
& p_{k, k}=\bar{s} p \bar{q}+s \bar{p} \bar{q}+s p q, \quad 2 \leq k \leq N-2 \\
& p_{k+1, k}=\bar{s} \bar{p} \bar{q}+\bar{s} p q+s \bar{p} q, \quad 2 \leq k \leq N-2 \\
& p_{k+2, k}=\bar{s} \bar{p} q, 2 \leq k \leq N-2 \\
& p_{N-1, N-1}=s p q+\bar{s} p \bar{q}+s \bar{p} \bar{q} \\
& p_{N-1, N}=s p \bar{q}+\bar{p} q \\
& p_{N, N-1}=\bar{s} \bar{p} \bar{q}+\bar{s} p q \\
& p_{N, N}=\bar{s} q+\bar{s} p \bar{q}+s \bar{p} \bar{q}
\end{aligned}
$$

The Chapman-Kolmogrov equations constructed for the system dynamics are as follows: 


$$
\begin{gathered}
\pi_{0}=(\bar{p}+p q) \pi_{0}+(\bar{s} \bar{p}+\bar{s} p q+s \bar{p} q) \pi_{1}+\bar{s} \bar{p} q \pi_{2} \\
\pi_{1}=p \bar{q} \pi_{0}+(\bar{s} p \bar{q}+s \bar{p} \bar{q}+s p q) \pi_{1}+(\bar{s} \bar{p} \bar{q}+\bar{s} p q+s \bar{p} q) \pi_{2}+ \\
+\bar{s} \bar{p} q \pi_{3} \\
\pi_{k}=s p \bar{q} \pi_{k-1}+(\bar{s} p \bar{q}+s \bar{p} \bar{q}+s p q) \pi_{k}+(\bar{s} \bar{p} \bar{q}+\bar{s} p q+s \bar{p} q) \pi_{k+1} \\
+\bar{s} \bar{p} q \pi_{k+2}, 2 \leq k \leq N-2 \\
\pi_{N-1}=s p \bar{q} \pi_{N-2}+(\bar{s} p \bar{q}+s \bar{p} \bar{q}+s p q) \pi_{N-1}+(\bar{s} \bar{p} \bar{q}+\bar{s} p q) \pi_{N} \\
\quad \pi_{N}=(s p \bar{q}+\bar{p} q) \pi_{N-1}+(s \bar{p} \bar{q}+\bar{s} p \bar{q}+\bar{s} q) \pi_{N}
\end{gathered}
$$

Multiplying (3) by $z^{k-1}$ and summing over $\mathrm{k}$ and using the normalizing condition $\sum_{k=0}^{N} \pi_{k}=1$, we get

$$
\begin{aligned}
{\left[z^{2}-(\bar{s}+s z)(\bar{p}+p z)(q+\bar{q} z)\right] g(z) } & =\left\{(1-\bar{s} p \bar{q}) z^{2}-(\bar{s}+s z)[\bar{p} \bar{q} z+q(\bar{p}+p z)]\right\} \pi_{1} \\
& -[\bar{s} \bar{p}(q+\bar{q} z)+q z(\bar{s} p+s \bar{p})] z \pi_{2}-\bar{s} \bar{p} q z^{2} \pi_{3} \\
& -s p \bar{q} z^{N} \pi_{N-2}-\left[(1-s p \bar{q} z-(\bar{s} p \bar{q}+s p q+s \bar{p} \bar{q})] z^{N} \pi_{N-1}\right. \\
- & -s p \bar{q} z-(\bar{s} p \bar{q}+s \bar{p} \bar{q}+s p q) z-(\bar{s} p q+s \bar{p} q+\bar{s} \bar{p} \bar{q})] z^{N} \pi_{N}
\end{aligned}
$$

Making use of eqs (1) and (2) in above equation, we have $\left[s p \bar{q} z^{2}-(1-s \bar{p} \bar{q}-s p q-p \bar{q}) z-\bar{s} \bar{p} q\right] g(z)=-\bar{s} \bar{p} q z \pi_{2}-[(\bar{s} \bar{p} \bar{q}+\bar{s} q+s \bar{p} q) z+\bar{s} \bar{p} q] \pi_{1}$

$$
\begin{aligned}
& -s p \bar{q} \pi_{N-2} z^{N}-[1-s p \bar{q} z-(\bar{s} p \bar{q}+s \bar{p} \bar{q}+s p q) z \\
& -\left[(1-s p \bar{q} z-(\bar{s} p \bar{q}+s p q+s \bar{p} \bar{q})] \pi_{N-1} z^{N}\right. \\
& -(\bar{s} p q+s \bar{p} q+\bar{s} \bar{p} \bar{q}) \pi_{N} z^{N}
\end{aligned}
$$

Now, the LHS of the eq. (7) has two roots, satisfying the condition such that $-1<z_{1}^{*}<0$ and $z_{2}^{*}>0$.

Substituting $z=z_{1}^{*}$ in eq. (7), we obtain

$$
\pi_{N}=\frac{\left(\begin{array}{c}
\bar{s} \bar{p} q z_{1}^{*} \pi_{2}+\left[(\bar{s} \bar{p} \bar{q}+\bar{s} q+s \bar{p} q) z_{1}^{*}+\bar{s} \bar{p} q\right] \pi_{1}+s p \bar{q} \pi_{N-2} z_{1}^{* N} \\
+\left[1-s p \bar{q} z_{1}^{*}-(s p q+\bar{s} p \bar{q}+s \bar{p} \bar{q}) z_{1}^{* N} \pi_{N-1}\right.
\end{array}\right)}{-\left[1-(s p q+\bar{s} p \bar{q}+s \bar{p} \bar{q}) z_{1}^{*}-(\bar{s} p q+s \bar{p} q+\bar{s} \bar{p} \bar{q}) z_{1}^{* N}\right.}
$$

Solving recursively, eqs (1)-(3) lead to the following relation 


$$
\pi_{1}=\xi \pi_{2}
$$

where,

$$
\xi=\frac{1}{c}\left((1-d)-\frac{c(b \cdot \beta+a c \cdot \alpha)}{\beta(1-d+c)-(c(1+b-d))}\right),
$$

for which

$$
a=\bar{s} \bar{p} q, b=\bar{s} \bar{p} \bar{q}+\bar{s} p q+s \bar{p} q, c=s p \bar{q}, d=s p q+\bar{s} p \bar{q}+s \bar{p} \bar{q}
$$

Also $\alpha$ and $\beta$ are given by

$$
\alpha=1-s \bar{p} \bar{q}, \quad \beta=1-s \bar{p} \bar{q}-d(1-s \bar{p} \bar{q})-a c
$$

Using the relation given in eq. (9) and then substituting the result obtained in (8), we have

$$
\pi_{N}=\frac{\left(a z_{1}^{*}+\left(b z_{1}^{*}+\bar{s} \bar{p} q\right) \xi\right) \pi_{2}}{\left[(d z+b-1)-c \cdot \alpha-\left(1-c z_{1}^{*}-d\right) \beta\right] z_{1}^{* N}}
$$

Again using eq. (4), we have

$$
g(z)=\frac{1}{\left(z-z_{2}^{*}\right)\left(z-z_{1}^{*}\right)}\left(\begin{array}{c}
-(a z+(b z+\overline{s p} q) \xi) \pi_{2} \\
-\frac{\left\{(c . \alpha+(1-c z-d) \beta+(1-d z-b))\left(\left[a z_{1}^{*}+\left(b z_{1}^{*}+\bar{s} \bar{p} q\right) \xi\right)\right\} \pi_{2}\right.}{\left((d z+b-1)-c \alpha-\left(1-c z_{1}^{*}-d\right) \beta\right)}\left(\frac{z}{z_{1}^{*}}\right)^{N}
\end{array}\right)
$$

where $z_{2}^{*}$ is the positive solution of the equation $s p \bar{q} z^{2}-(1-s \bar{p} \bar{q}-s p q-p \bar{q}) z-\bar{s} \bar{p} q$.

Using eq. (1) and after some simple algebraic manipulation, we get $g(z)=\frac{1}{\left(z-z_{2}^{*}\right)\left(z-z_{1}^{*}\right)}\left(\begin{array}{l}-(a z+(b z+\bar{s} \bar{p} q) \xi) \theta \pi_{0} \\ \left.-\frac{\left\{(c . \alpha+(1-c z-d) \beta+(1-d z-b))\left(\left[a z_{1}^{*}+\left(b z_{1}^{*}+\bar{s} \bar{p} q\right) \xi\right)\right\}\right.}{\left((d z+b-1)-c \alpha-\left(1-c z_{1}^{*}-d\right) \beta\right.}\left(\frac{z}{z_{1}^{*}}\right)^{N}\right)\end{array}\right.$

where $\theta=\frac{p \bar{q} \cdot \pi_{0}}{a+(\bar{s} \bar{p}+\bar{s} p q+s \bar{p} q) \xi}$.

\subsection{RCE- Immune Service Killing Policy}

In case of immune service killing policy, the customers in service are immune from killing by the negative arrivals. Thus according to this killing discipline a customer currently in service is not allowed to be 
removed by a negative arrival, i.e. the customer which is taking the service is immune from killing and this killing discipline is itself called as the immune- killing servicing.

The one-step transition probabilities for this case are given by:

$$
\begin{aligned}
& p_{0,0}=\bar{p} \\
& p_{1,0}=\bar{s} \bar{p} \\
& p_{0,1}=p \\
& p_{3,1}=\bar{s} \bar{p} \\
& p_{1,1}=\bar{s} p+s \bar{p}+s p q \\
& p_{2,1}=\bar{s} \bar{p}+\bar{s} p q+s \bar{p} q \\
& p_{k-1, k}=s p \bar{q}, \quad 2 \leq k \leq N-2 \\
& p_{k, k}=\bar{s} p \bar{q}+s \bar{p} \bar{q}+s p q, 2 \leq k \leq N-2 \\
& p_{k+1, k}=\bar{s} \bar{p} \bar{q}+\bar{s} p q+s \bar{p} q, 2 \leq k \leq N-2 \\
& p_{k+2, k}=\bar{s} \bar{p} q, 2 \leq k \leq N-2 \\
& p_{N-1, N-1}=\bar{s} p+s \bar{p}+s p q \\
& p_{N-1, N}=p \\
& p_{N, N-1}=\bar{s} \bar{p}+s \bar{p} q \\
& p_{N, N}=s \bar{p} \bar{q}
\end{aligned}
$$

The Chapman-Kolmogrov equations are given by:

$$
\begin{gathered}
\pi_{0}=\bar{p} \pi_{0}+\bar{s} \bar{p} \pi_{1} \\
\pi_{1}=p \pi_{0}+(\bar{s} p+s \bar{p}+s p q) \pi_{1}+(\bar{s} \bar{p}+\bar{s} p q+s \bar{p} q) \pi_{2}+\bar{s} \bar{p} q \pi_{3} \\
\pi_{k}=s p \bar{q} \pi_{k-1}+(\bar{s} p \bar{q}+s \bar{p} \bar{q}+s p q) \pi_{k}+(\bar{s} \bar{p} \bar{q}+\bar{s} p q+s \bar{p} q) \pi_{k+1} \\
+\bar{s} \bar{p} q \pi_{k+2}, 2 \leq k \leq N-2 \\
\pi_{N-1}=s p \bar{q} \pi_{N-2}+(\bar{s} p+s \bar{p} \bar{q}+s p q) \pi_{N-1}+(\bar{s} \bar{p}+s \bar{p} q) \pi_{N} \\
\pi_{N}=p \pi_{N-1}+s \bar{p} \bar{q} \pi_{N}
\end{gathered}
$$


Now, multiplying (16) by $z^{k-1}$ and summing over $\mathrm{k}$ and using the normalizing condition $\sum_{k=0}^{N} \pi_{k}=1$, we get

$$
\begin{aligned}
{\left[z^{2}-(\bar{s}+s z)(\bar{p}+p z)(q+\bar{q} z)\right] } & g(z)=\left\{(1-\bar{s} p \bar{q}) z^{2}-(\bar{s}+s z)[\bar{p} \bar{q} z+q(\bar{p}+p z)]\right\} \pi_{1} \\
& -[\bar{s} \bar{p}(q+\bar{q} z)+q z(\bar{s} p+s \bar{p})] z \pi_{2}-\bar{s} \bar{p} q z^{2} \pi_{3}-s p \bar{q} \pi_{N-2} z^{N} \\
& -\left[(1-s p \bar{q} z-(\bar{s} p \bar{q}+s p q+s \bar{p} \bar{q})] \pi_{N-1} z^{N}\right. \\
& -\left[1-s p \bar{q} z-(\bar{s} p \bar{q}+s \bar{p} \bar{q}+s p q) z-(\bar{s} p q+s \bar{p} q+\bar{s} \bar{p} \bar{q}) \pi_{N} z^{N}\right.
\end{aligned}
$$

Making use of eqs (14) and (15) in above equation, we have $\left[s p \bar{q} z^{2}-(1-s \bar{p} \bar{q}-s p q-p \bar{q}) z-\bar{s} \bar{p} q\right] g(z)=-\bar{s} \bar{p} q z \pi_{2}-[(\bar{s} \bar{p} \bar{q}+\bar{s} q+s \bar{p} q) z+\bar{s} \bar{p} q] \pi_{1}-s p \bar{q} \pi_{N-z^{2}}$

$$
\begin{aligned}
& -\left[(1-s p \bar{q} z-(\bar{s} p \bar{q}+s p q+s \bar{p} \bar{q})] \pi_{N-1} z^{N}\right. \\
& -\left[1-s p \bar{q} z-(\bar{s} p \bar{q}+s \bar{p} \bar{q}+s p q) z-(\bar{s} p q+s \bar{p} q+\bar{s} \bar{p} \bar{q}) \pi_{N} z^{N}\right.
\end{aligned}
$$

The LHS of the eq. (20) has two roots, satisfying the condition $-1<z_{1}^{*}<0$ and $z_{2}^{*}>0$.

Substituting $z=z_{1}^{*}$ in eq. (20), we obtain

$$
\pi_{N}=\frac{\left(\begin{array}{c}
\bar{s} \bar{p} q z_{1}^{*} \pi_{2}+\left[(\bar{s} \bar{p} \bar{q}+\bar{s} q+s \bar{p} q) z_{1}^{*}+\bar{s} \bar{p} q\right] \pi_{1}+s p \bar{q} \pi_{N-2} z_{1}^{* N} \\
+\left[1-s p \bar{q} z_{1}^{*}-(s p q+\bar{s} p \bar{q}+s \bar{p} \bar{q}) z_{1}^{* N} \pi_{N-1}\right.
\end{array}\right)}{-\left[1-(s p q+\bar{s} p \bar{q}+s \bar{p} \bar{q}) z_{1}^{*}-(\bar{s} p q+s \bar{p} q+\bar{s} \bar{p} \bar{q}) z_{1}^{* N}\right.}
$$

Now, using the relation given in (9), and inserting the values from eqs (17) and (18) in (21), we find

$$
\pi_{N}=\frac{\left(a z_{1}^{*}+\left(b z_{1}^{*}+\bar{s} \bar{p} q\right) \xi\right) \pi_{2}}{\left[(d z+b-1)-c \cdot \alpha^{\prime}-\left(1-c z_{1}^{*}-d\right) \beta^{\prime}\right] z_{1}^{* N}}
$$

where $\alpha^{\prime}$ and $\beta^{\prime}$ are given by

$$
\alpha^{\prime}=\left(\frac{\left(1-d^{\prime}\right)\left(1-f^{\prime}\right)-e^{\prime} p}{c \cdot p}\right), \quad \beta^{\prime}=\left(\left(1-f^{\prime}\right) / p\right.
$$

such that $d^{\prime}=(\bar{s} p+s \bar{p} \bar{q}+s p q), e^{\prime}=(\bar{s} \bar{p}+s \bar{p} q)$ and $f^{\prime}=s \bar{p} \bar{q}$.

Again preceding the analysis in the same manner as done in the previous case (i. e. in-immune service killing), we finally obtained the probability generating function as 
$g(z)=\frac{1}{\left(z-z_{2}^{*}\right)\left(z-z_{1}^{*}\right)}\left(\begin{array}{l}-(a z+(b z+\bar{s} \bar{p} q) \xi) \theta^{\prime} \pi_{0} \\ -\frac{\left\{\left(c \cdot \alpha^{\prime}+(1-c z-d) \beta^{\prime}+(1-d z-b)\right)\left(\left[a z_{1}^{*}+\left(b z_{1}^{*}+\bar{s} \bar{p} q\right) \xi\right)\right\} \theta^{\prime} \pi_{0}\right.}{\left((d z+b-1)-c \alpha^{\prime}-\left(1-c z_{1}^{*}-d\right) \beta^{\prime}\right)}\left(\frac{z}{z_{1}^{*}}\right)^{N}\end{array}\right)$

Where $\theta^{\prime}=\frac{1}{s \xi}$

\section{Performance Measures}

In this section some performance measures in terms of the stationary distribution are derived

$>$ The probability generating function of the number of customers in the waiting line is given by:

$$
\psi(z)=\pi_{0}+g(z)
$$

$>$ The average number of customers in the system is given by

$$
E(S)=\sum_{k=o}^{N} k \pi_{k}
$$

$>$ The average number of customers in the queue is given as

$$
E(Q)=\sum_{k=1}^{N}(k-1) \pi_{k}
$$

Blocking Probability of the system is given by

$$
\mathrm{P}(\mathrm{B})=\pi_{N}
$$

\section{Numerical Results}

In this section, we discuss the neurro-fuzzy approach and obtain the numerical results for various performance measures. The effects of the parameters $\mathrm{p}, \mathrm{q}$ and $\bar{s}$ on the stationary probabilities $\pi_{k}, 0 \leq k \leq N$ have also been shown. The graphs are depicted for both the two policies, i. e. immune and in-immune servicing policies.

For computing the performance measures, the Artificial NeuroFuzzy Inference System (ANFIS) network is built by using the fuzzy toolbox of real time systems in MATLAB 6.5. It is worth-mentioning 
that soft computing approach provides flexible information processing capabilities for handling real life situations. That's why it's a very useful technique to solve complex problems. Neuro-fuzzy techniques are the most powerful soft computing techniques and can also be employed for performance prediction of queueing models. This approach is applied by varying the arriving probability $q$, of the negative customer. Here ' $q$ ' is considered as the linguistic variable for fuzzy systems and is also considered as the input parameter. The gaussian function is used for describing the membership function of the input parameter q. The linguistic values for $\mathrm{q}$ are given in Table 1 . Further, the shapes of the membership functions are illustrated in Fig. 3 (a) and 4 (a), respectively. In Fig. 3 (b) and 4 (b), the smooth lines depict the values for analytical results and the broken lines correspond to the approximated results obtained by the neurro fuzzy technique.

We present the numerical results for various performance measures. For this purpose, we have chosen the system parameters as $\bar{s}=0.8, \mathrm{p}=0.3, \mathrm{~N}=7$. Tables $2-4$ show the effects of $\mathrm{p}, \mathrm{q}$ and $\bar{s}$ on $\pi_{k}$ for in-immune service killing policy. From Tables 2 and 3, it is seen that for the increasing values of $\mathrm{k}$, the stationary probabilities $\left(\pi_{k}\right)$ tend to decrease for both $\mathrm{p}$ and $\mathrm{q}$, respectively. Also $\pi_{0}$ decreases (increases) for increasing values of $p(q)$. This implies that with more positive arrivals, there is lower probability of the system to become empty. On the contrary, if more negative arrivals join the queue then the system will be more prone to be empty. The same decreasing pattern of $\pi_{k}$ is observed for the increasing values of $\mathrm{k}$ by varying $\bar{s}$ in Table 4 . Also, with the increasing values of $\bar{s}$, there is a decrement in $\pi_{k}$. This illustrates the fact that the increase in probability of service oftenly tends to decrease in the number of customers in the system.

Table 1. Linguistic values for the input parameters.

\begin{tabular}{|c|c|c|c|}
\hline $\begin{array}{c}\text { Input variable } \\
(\mathbf{q})\end{array}$ & $\begin{array}{c}\text { Membership functions } \\
\text { for q }\end{array}$ & $\begin{array}{c}\text { Shapes of membership } \\
\text { functions }\end{array}$ & Linguistic Values \\
\hline $\begin{array}{c}\text { For evaluating } \pi_{0} \\
\text { (Fig. 3 (b)) }\end{array}$ & 4 & Gaussian & : Very low \\
\hline For evaluating $\pi_{1}$ & & (Fig. 3 (a)) & Average \\
(Fig. 4 (b)) & 3 & $\begin{array}{c}\text { Gaussian } \\
\text { (Fig. 4 (a)) }\end{array}$ & $\begin{array}{l}\text { - High } \\
\text { - High }\end{array}$ \\
\hline
\end{tabular}


Table 2. Stationary probabilities $\pi_{k}$ for the varying values of $p$.

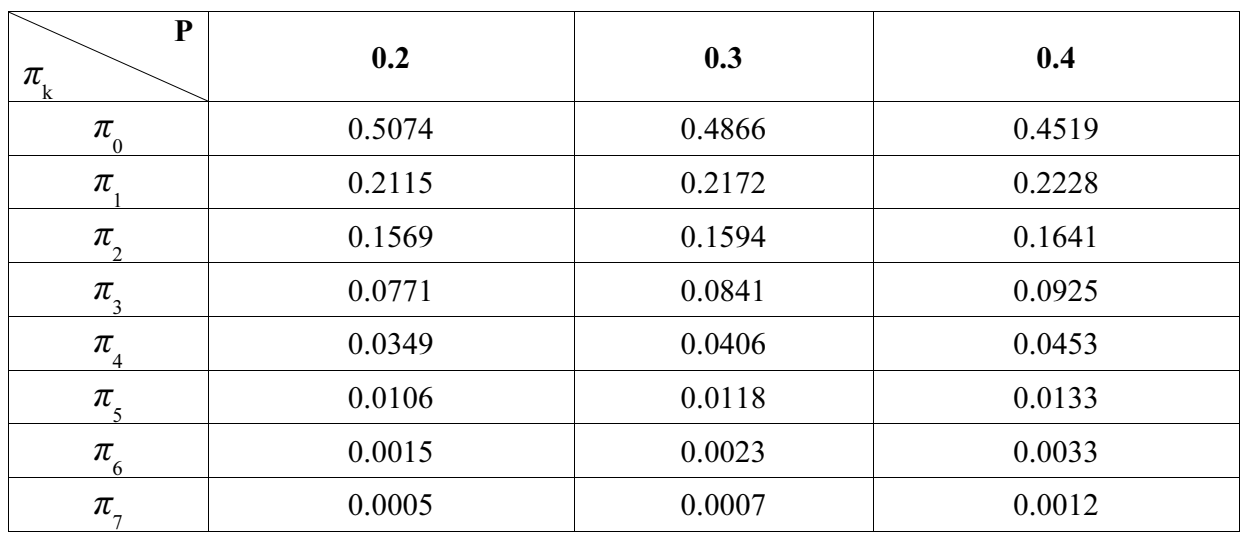

Table 3. Stationary probabilities $\pi_{k}$ for the varying values of $q$.

\begin{tabular}{|c|c|c|c|}
\hline$\pi_{\mathrm{k}} \mathbf{q}$ & $\mathbf{0 . 2}$ & $\mathbf{0 . 3}$ & $\mathbf{0 . 4}$ \\
\hline$\pi_{0}$ & 0.4976 & 0.5397 & 0.5794 \\
\hline$\pi_{1}$ & 0.2141 & 0.2163 & 0.2181 \\
\hline$\pi_{2}$ & 0.1466 & 0.1467 & 0.1475 \\
\hline$\pi_{3}$ & 0.0711 & 0.0743 & 0.0783 \\
\hline$\pi_{4}$ & 0.0319 & 0.0335 & 0.0356 \\
\hline$\pi_{5}$ & 0.0082 & 0.0084 & 0.0087 \\
\hline$\pi_{6}$ & 0.0014 & 0.0018 & 0.0022 \\
\hline$\pi_{7}$ & 0.0016 & 0.0019 & 0.0023 \\
\hline
\end{tabular}

Table 4. Stationary probabilities $\pi_{k}$ for the varying values of $\bar{s}$.

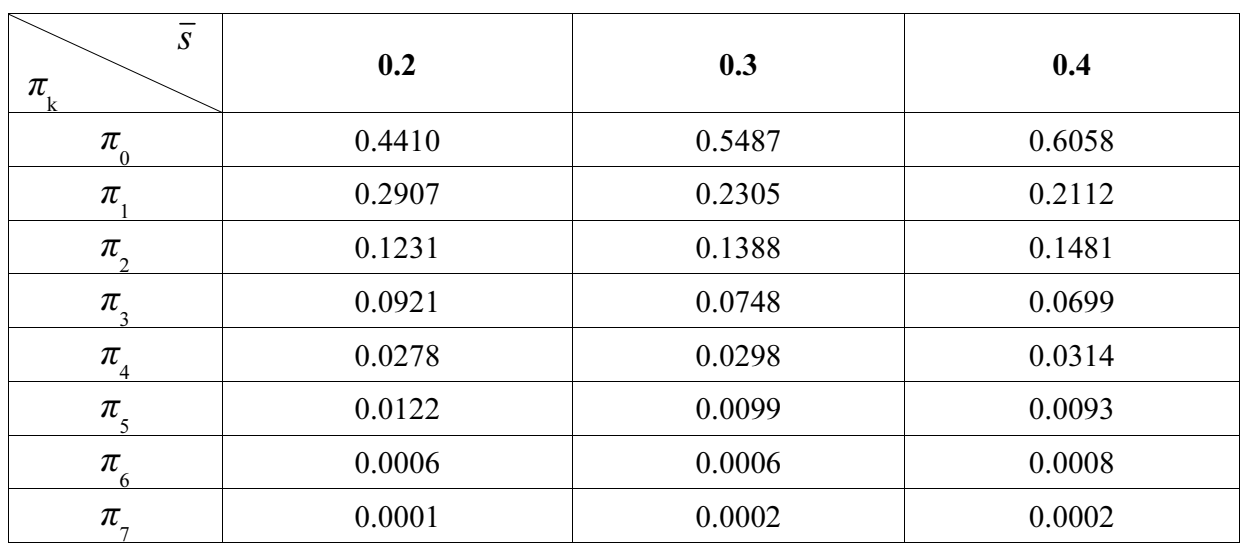


Figures 1(a-c) and 2 (a-c) depict the results for the system length and the queue length for different values of $\mathrm{N}$ by varying $\mathrm{p}, \mathrm{q}$ and $\bar{s}$, respectively for in-immune service killing policy. The default parameters are set as $\mathrm{p}=0.35, \mathrm{q}=0.8, \bar{s}=0.9$. Figures $1(\mathrm{a}-\mathrm{c})$ and 2 (a-c) exhibit the varying trends of system length $\mathrm{E}[\mathrm{S}]$ and the queue length $\mathrm{E}[\mathrm{Q}]$, respectively. It is observed that both $\mathrm{E}[\mathrm{S}]$ and $\mathrm{E}[\mathrm{Q}]$ go on increasing for the increasing values of $\mathrm{N}$, i.e. capacity of the system. Thus it illustrates the fact that an increasing capacity of the system tends to increase in the system length and the queue length, which is in agreement with the realistic situation. Also from Fig. 1(a-b) and $2(a-b)$, it is seen that both $E[S]$ and $E[Q]$ increase sharply for the increasing values of $p$ and $q$, respectively. However, it is noticed that the values of $E[Q]$ are quite lesser than those of $E[S]$ for varying values of $p$ and $q$ in each case because the system size is always greater than queue size which is quite obvious. On the contrary, Fig. 1(c) and 2 (c) show the decreasing trends of $\mathrm{E}[\mathrm{S}]$ and $\mathrm{E}[\mathrm{Q}]$ for different values of $\bar{s}$, which also agrees with the realistic situation wherein the increased service rate tends to decrease in the system size or queue size.

Figure 3 (b) displays the effect of parameter q on the stationary probabilities $\pi_{0}$ for both the immune and in-immune cases, respectively. It is observed that $\pi_{0}$ is an increasing function of $\mathrm{q}$ as more negative arrivals will lead to the increasing probability of the system to be empty, which is quite obvious. It is also seen that this probability is smaller for the immune case as compared to the in-immune case. This illustrates the fact that when the customers in service are immune from killing, it leads to the lower probability of the system to become empty and vice versa. In the contrast, Fig. 4 (b) shows the reversed effect of the parameter q on the probability $\pi_{1}$ for immune and in-immune cases. It is noted that $\pi_{1}$ has the higher values for the immune case whereas it has the lesser values for in-immune case. Moreover, the results displayed for immune and inimmune cases are at par with the neurro-fuzzy results, for this, 'q' is considered as the linguistic variable (i.e. fuzzy variable). 


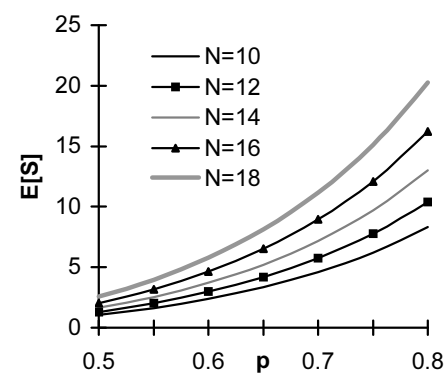

(a)

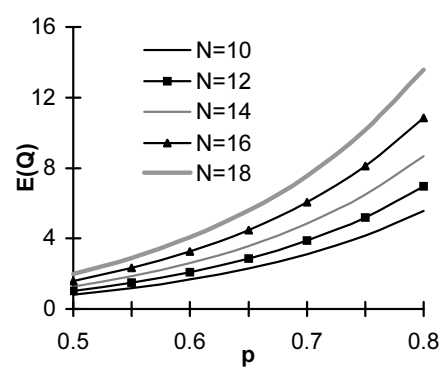

(a)
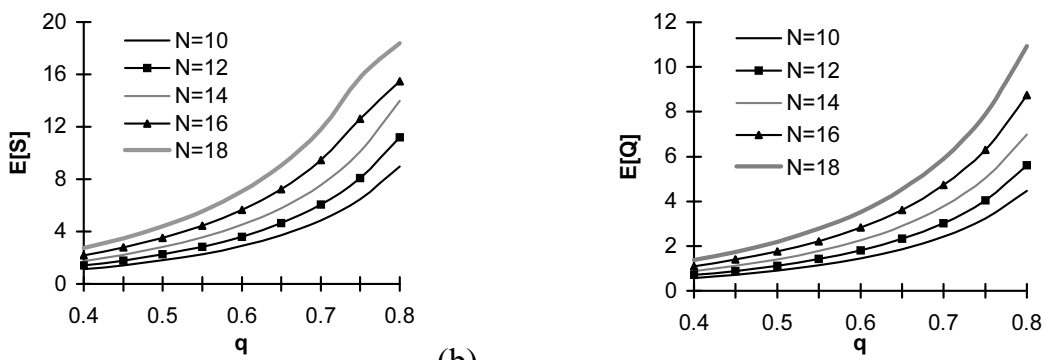

(b)
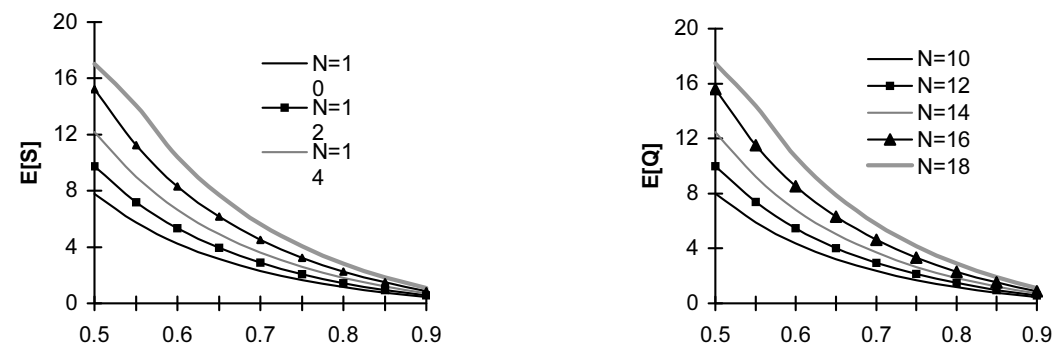

$\bar{S}(\mathrm{c})$

$\bar{s}(\mathrm{c})$

Fig. 1 (a-c): Expected system length $v s$ (a) p (b) q and (c) $\bar{S}$
Fig. 2 (a-c): Expected queue length vs

(a) p (b) q and (c) $\bar{s}$

Overall we conclude that the results obtained for system size and the queue size describe their increasing behavior with respect to parameters $\mathrm{p}$ and $\mathrm{q}$. Also the influence of increment in parameter $\bar{s}$ is such that the system length and the queue length both show the decreasing pattern. Finally, we explore that the approximate values of the results by using ANFIS, which are quite closer to those obtained analytically. 


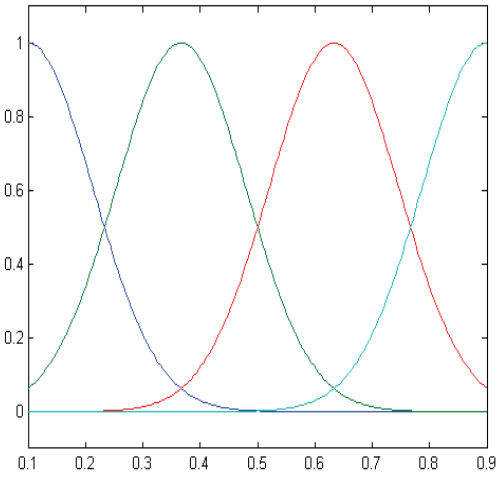

(a)

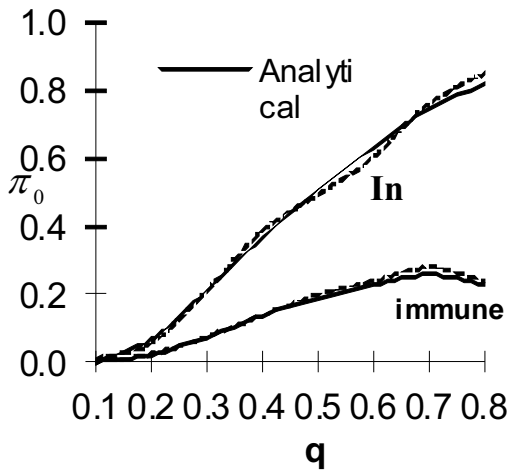

(b)

Fig. 3. Graphs depicting (a) the shapes of the membership functions for the arrival Rate (q) of the positive customers (b) the effect of $q$ on the probability $\pi_{0}$.

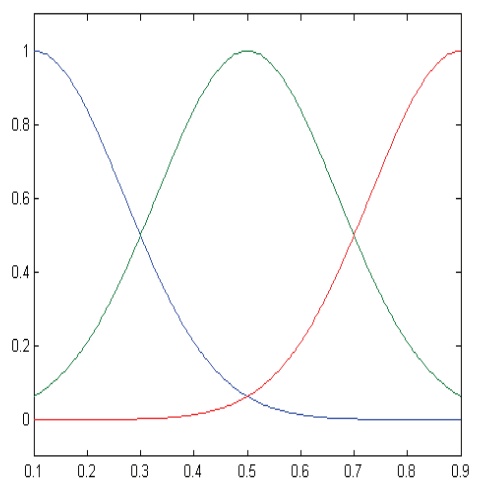

(a)

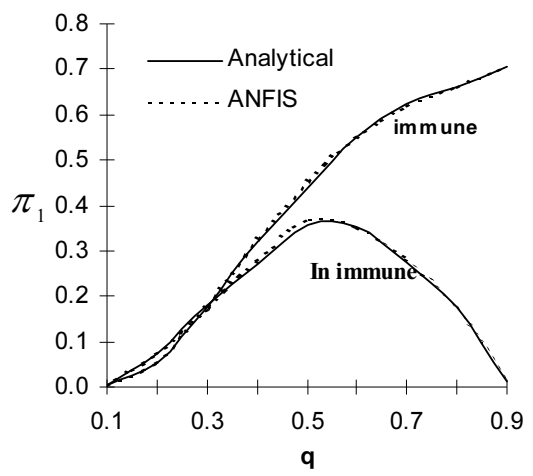

(b)

Fig. 4. Graphs depicting (a) the shapes of the membership functions for the arrival Rate (q) of the positive customers (b) the effect of $q$ on the probability $\pi_{1}$.

\section{Discussions}

The discrete time queueing models have wide range of applicability in the real time digital computer and communication systems including BISDN system, wherein various protocols are implemented in which the transport mechanism is done by packetization. In this paper, we have considered the discrete time $\mathrm{Geo} / \mathrm{Geo} / 1$ finite capacity queueing system 
with both positive and negative arrival stream. The analysis is done by taking into consideration the two types of service killing discipline, RCE in-immune and immune policy, according to which the customer currently being served can and cannot be killed by the negative customer, respectively. The in-immune killing discipline can be suited to model the killing signals in speculative parallelism whereas the immune policy can be used to model the load balancing system in which the work is transferred from the overloaded queues.

The main contribution of the present study is to analyze a finite capacity discrete time model of a queueing system with both types of the customers. Such discrete time queueing model is appropriate particularly to describe the varied queueing related phenomena in the mobile and computer and communication networks wherein the time axis is slotted in the discrete intervals of time, e.g. in Time Division Multiplexing Access (TDMA). The stationary probability distribution and many performance measures are derived in terms of the generating functions. We have also employed the neurro fuzzy approach for computing the performance measures using the fuzzy toolbox of the MATLAB software. It is seen that the results that are obtained by the neurro fuzzy technique are quite closer to the analytical results obtained by employing the successive over relaxation (SOR) method. The neurro fuzzy controller developed facilitates an easier and fast solution to real time queueing systems where it is difficult to obtain analytical results.

\section{References}

[1] Gelenbe, E., Random neural networks with negative and positive signals and product form solution, Neural Comput., 1: 502-510 (1989).

[2] Harrison, P.G. and Pitel, E., Performance Modeling of Communication Networks and Computer Architectures, Second edition, Addison Wesley, New York (1993).

[3] Gelenbe, E., Product form queueing network with negative and positive customers, J. Appl. Prob., 28: 565-663 (1991).

[4] Gelenbe, E., Learning in the recurrent random neural network, Neural Comput., 5: 154-164 (1993a).

[5] Gelenbe, E., G-networks: A unifying model for neural and queueing networks, Ann. Oper. Res., 48: 433-461(1994).

[6] Gelenbe, E., The first decade of G-networks, Euro. J. Oper. Res., 126: 231-232 (2000).

[7] Artalejo, J. R., G-networks: A versatile approach for work removal in queueing networks, Euro. J. Oper. Res., 126: 233-249 (2000).

[8] Shin, Y.W. and Choi, B.D., A queue with positive and negative arrivals governed by a Markov chain, Cambridge University Press, 17: 407-501 (2003).

[9] Zhu, Y. and Zhang, Z., M/GI/1 queues with services of both positive and negative customers, J. Appl. Prob., 41: 1157-1170 (2004). 
[10] Wang, J. and Zhang, P., Discrete time retrial queue with negative customers and unreliable server, Comp. Indust. Engg., 56(4): 1216-1222 (2009).

[11] Chae, K.C., Park, H.M. and Yang, W.S., A GI/Geo/1 queue with negative and positive customers, App. Math. Model., 34(6): 1662-1671 (2010).

[12] Chakka, R. and Harrison, P.G., A Markov modulated multi-server queue with negative customers-the MMCPP/ GE / c / LG-queue, Acta Inform., 37: 881-919 (2001).

[13] Harrison, P.G. and Pitel, E., Sojourn times in single-server queues with negative customer, J. Appl., Prob., 30: 943-963 (1993).

[14] Atenica, I. and Moreno, P., A single-server G-queues in discrete-time with geometrical arrival and service process, Perf. Eval., 59: 85-97 (2005).

[15] Bruneel, H. and Kim, B.G., Discrete-time Models for Communication Systems Including ATM, Kluwer Academic Publishers, Boston (1993).

[16] Artalejo, J.R., Atenica, I. and Moreno, P., A discrete time $G e 0{ }^{[\mathrm{X}]} / \mathrm{G} / 1$ retrial queue with control of admission, Appl. Math. Model., 29(11): 1100-1120 (2005).

[17] Ciaramella, A. Tagliaferri, R., Pedrycz, W. and Nola, A.D., Fuzzy relational neural network, Int. J. Approx. Reason., 41(2): 146-163 (2006).

[18] Kaedi, M., Movahhedinia, N. and Jamshidi, K., Traffic signal timing using two dimensional correlation, neurro-fuzzy and queueing based neural networks, Neural Computing and Applications, Publisher Springer London,1433-3058 (2007). 


\section{صفوف الانتظار Geo/Geo/1/N مع قواعد المناعة والخدمة المانعة للاستبعاد}

\section{مادهي جان *، وجي سي شارما * وسابنا شاكراوارتي * *

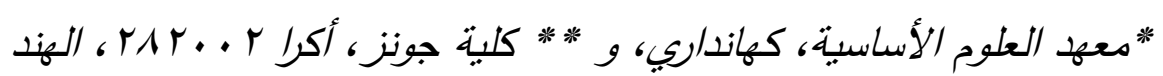

السستخلص. البحث الحالي هو دراسـة صفوف انتظار متفرد الوقت

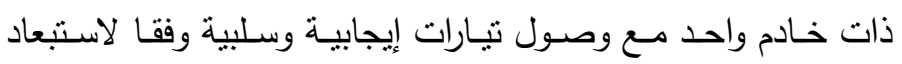

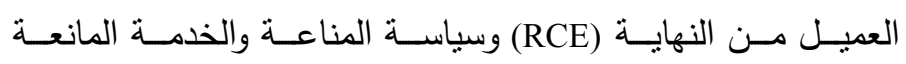
للاستبعاد. هذه الدراسـة هى تعميم لصفوف الانتظـار ذات العمـلاء

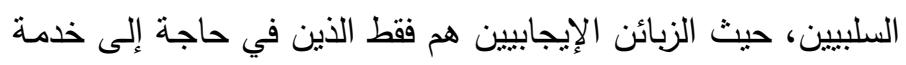

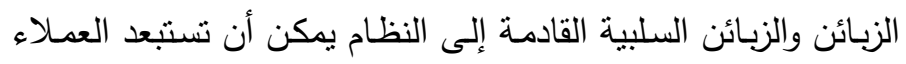

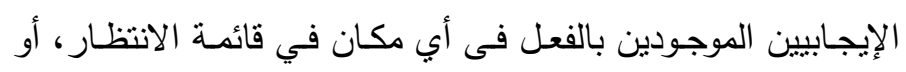

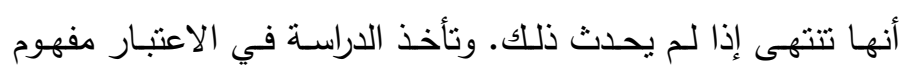

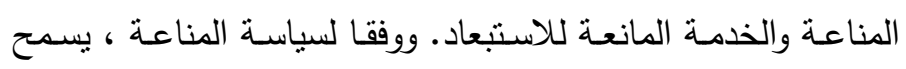

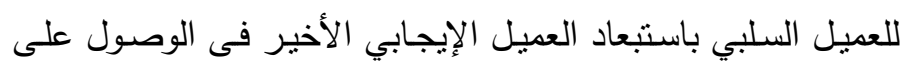

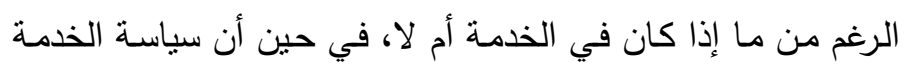

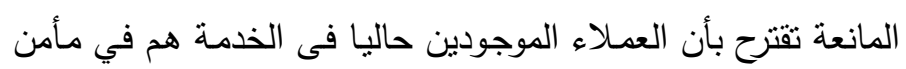

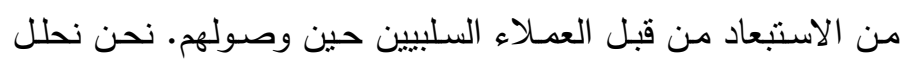
طابورًا من الوافدين وفق الوصـول كدالـة هندسية لكل مـن العملاء

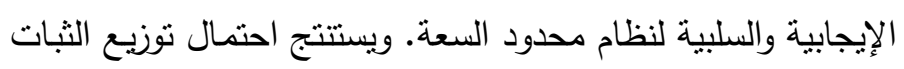

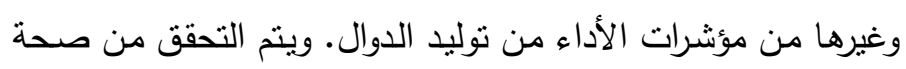

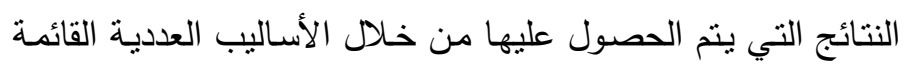

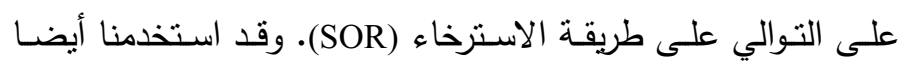


الـهـج العصبـى الغـامض لإظهار النتـائج التقريبيـة لمقاييس الأداء المختلفة.

الكلمات الدفتاحية: مجموعة قائمة الانتظار ، المتقطعة الوقت، محدود السعة، الوصول السلبي، المناعة والخدمة المانعة

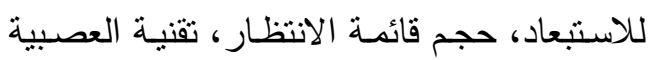

الغامضة. 